\title{
Immunothérapie et tube digestif Introduction
}

\section{T. Aparicio}

(C) Lavoisier SAS 2015

Le système immunitaire est une des mécaniques les plus complexes du corps humain.

La connaissance de celui-ci a beaucoup évolué ces dernières années et des thérapeutiques ciblées agissant directement sur certains de ses acteurs sont maintenant disponibles.

En pathologies digestives, le système immunitaire a un rôle-clé dans deux principales situations : les maladies inflammatoires chroniques de l'intestin (MICI) et les cancers.

Dans le cas des MICI, la dérégulation du système immunitaire intervient très tôt dans le développement de la maladie. La réponse inflammatoire agressive va ensuite être la cause des lésions digestives. Les traitements immunosuppresseurs non spécifiques sont utilisés depuis très longtemps dans le traitement des MICI. Puis les anticorps monoclonaux dirigés contre le TNF- $\alpha$ ont révolutionné la prise en charge des patients. D'autres immunothérapies plus spécifiques encore sont attendues et devraient permettre de nouveaux progrès.

Dans le cancer colorectal (CCR), le système immunitaire joue son rôle en contenant la progression tumorale au début du processus. Puis, il perd de son efficacité et les cellules tumorales acquièrent la capacité de le rendre inefficace. Depuis plus de 10 ans des anticorps monoclonaux dirigés contre certains facteurs de croissance (EGFR, VEGF) ont permis une avancée majeure dans le traitement des patients atteints de cancer colorectal métastatique. Il s'agissait déjà d'une immunothérapie, mais dite passive car la cible n'était pas directement le système immunitaire. Aujourd'hui nous sommes peut-être à la veille d'une nouvelle révolution thérapeutique avec l'apparition de traitements ciblant directement les acteurs du système immunitaire afin de le rendre à nouveau efficace en situation de cancer avancé.

Dans ces deux grands champs de la pathologie digestive que sont les MICI et les cancers, la connaissance des mécanismes physiopathologiques a été à l'origine du développement de nouvelles stratégies thérapeutiques. Ce dossier est organisé en deux volets pour chacune de ces pathologies, l'un traitant de la physiopathologie et l'autre de la thérapeutique.

Bonne lecture

T. Aparicio $(\bowtie)$

Service de Gastroentérologie et Cancérologie Digestive, Hôpital Avicenne, HUPSSD, APHP, 125, rue de Stalingrad, Bobigny, Université Paris 13, Sorbonne Paris-Cité

e-mail : thomas.aparicio@avc.aphp.fr 